\title{
Influence of trapped electrons on ECCD in Heliotron J
}

\section{$\operatorname{AUTHOR}(\mathrm{S})$ :}

Nagasaki, K.; Yamamoto, S.; Yoshino, H.; Sakamoto, K.; Marushchenko, N.B.; Turkin, Y.; Mizuuchi, T.; ... Cappa, Á.; Blackwell, B.D.; Sano, F.

\section{CITATION:}

Nagasaki, K.... [et al]. Influence of trapped electrons on ECCD in Heliotron J. Nuclear Fusion 2011, 51(10): 103035.

\section{ISSUE DATE:}

2011-09

URL:

http://hdl.handle.net/2433/147976

\section{RIGHT:}

(C) 2011 IAEA, Vienna; This is not the published version. Please cite only the published version.; この論文は出版社版でありません。引用の際に は出版社版をご確認ご利用ください。 


\title{
Influence of Trapped Electrons on ECCD in Heliotron J
}

\author{
K. Nagasaki ${ }^{1)}$, S. Yamamoto ${ }^{1)}$, H. Yoshino ${ }^{2)}$, K. Sakamoto ${ }^{1)}$, N. B. Marushchenko ${ }^{3)}$, \\ Y. Turkin ${ }^{3)}$, T. Mizuuchi ${ }^{1)}$, H. Okada ${ }^{1)}$, K. Hanatani ${ }^{1)}$, T. Minami $^{1}{ }^{1}$, K. Masuda $^{1)}$, \\ S. Kobayashi ${ }^{1)}$, S. Konoshima ${ }^{1)}$, M. Takeuchi ${ }^{1}$, Y. Nakamura ${ }^{2)}$, S. Ohshima ${ }^{4}$, K. Mukai ${ }^{2}$, \\ H. Y. Lee ${ }^{2)}$, K. Mizuno ${ }^{2)}$, Y. Yoshimura ${ }^{5)}$, G. Motojima ${ }^{5)}$, Á. Cappa ${ }^{6)}$, B. D. Blackwel1 ${ }^{7)}$, F. Sano ${ }^{1)}$ \\ 1) Institute of Advanced Energy, Gokasho, Uji, Kyoto University, Japan \\ 2) Graduate School of Energy Science, Gokasho, Uji, Kyoto University, Japan \\ 3) Max-Planck-Institut für Plasmaphysik, IPP-EURATOM Association, D-17491, Greifswald, \\ Germany \\ 4) Kyoto Univ. Pioneering Research Unit for Next Generation, Gokasho, Uji, Japan \\ 5) National Institute for Fusion Science, Toki, Japan \\ 6) Laboratorio Nacional de Fusión, EURATOM-CIEMAT, Spain \\ 7) Plasma Research Laboratory, Australian National University, Canberra, Australia \\ E-mail contact of main author: nagasaki@iae.kyoto-u.ac.jp
}

\begin{abstract}
Second-harmonic electron cyclotron current drive (ECCD) experiments have been performed in the stellarator/heliotron device $(\mathrm{S} / \mathrm{H})$, Heliotron J. A focused Gaussian beam is injected with the parallel refractive index, $N_{\|}$, ranging from -0.05 to 0.6 . The EC driven current is estimated by excluding the bootstrap current from the total current. The experimental results show that the EC driven current is determined by the local magnetic field structure where the EC power is deposited. The maximum EC driven current is attained around $N_{\|}=0.5$ when the EC power is deposited at nearly the top of the magnetic ripple. A large increase in electron cyclotron emission (ECE) signals is observed when the EC current is driven, indicating an important role for high-energy electrons in the ECCD. The experimental results for $N_{\|}$and $B$ dependence agree with a ray tracing simulation using a parallel momentum conservation model in which the trapped particle effect is included.
\end{abstract}




\section{Introduction}

Noninductive current plays an important role in the realization of high-performance plasmas and the sustainment of steady-state plasmas in toroidal fusion devices. In stellarator/heliotron $(\mathrm{S} / \mathrm{H})$ systems, no Ohmic current is required for equilibrium since the confinement magnetic field is generated by external coils. However, it is known that noninductive current flows in $\mathrm{S} / \mathrm{H}$ systems as well as in tokamaks. Finite plasma pressure gradient drives bootstrap current, and tangential neutral beam injection (NBI) generates a noninductive NB current. Both bootstrap and NB currents modify the rotational transform profile, thereby affecting the equilibrium and stability. In the $\mathrm{S} / \mathrm{H}$ device, Heliotron J, we have demonstrated that magnetohydrodynamic (MHD) activity of the $m / n=2 / 1$ mode ( $m$ and $n$ are the poloidal and toroidal mode numbers, respectively) is excited when the rotational transform increases from 0.48 to 0.50 by bootstrap current and NB driven current [1]. The noninductive current also modifies the edge field topology and divertor performance [2]. Furthermore, a transition onset to an improved confinement mode in NBI plasmas has been observed in relation to the noninductive current in the Heliotron $\mathrm{J}$ [3].

Electron cyclotron current drive (ECCD) is recognized as being a useful scheme for stabilizing MHD instabilities in tokamaks[4, 5]. In S/H systems, ECCD is expected to be an effective current drive scheme to suppress the noninductive current and to tailor the rotational transform profile, particularly in low-shear devices. An ECCD experiment in the Heliotron J has shown that the EC driven current strongly depends on the magnetic field configuration [6,7], suggesting that ECCD is determined by the balance between the Fisch-Boozer effect [8] and the Ohkawa effect [9]. We also demonstrated that a net zero current state was maintained by cancelling the bootstrap current with ECCD. From the viewpoint of diagnostics, S/H systems have the advantage of allowing precise measurement of the EC driven current. Because no Ohmic current is required in $\mathrm{S} / \mathrm{H}$ systems, they achieve an accuracy of $0.1 \mathrm{kA}$ using conventional Rogowski coils. Comparing experimental results between tokamaks and helical systems gives us a deeper understanding of the ECCD physical mechanism in toroidal devices. Experimental research on ECCD has been performed in the Heliotron J, W7-AS [10], TJ-II [11], CHS [12] and LHD [13]. Comparative studies among various helical devices have also been performed under international collaboration, and the ECCD efficiency was found to be similar [14].

We have previously launched a non-focused Gaussian beam with a fixed angle for electron cyclotron heating $(\mathrm{ECH}) / \mathrm{ECCD}$ in the Heliotron J. Although several experimental ECCD findings have been obtained using this launcher, the system has limitations for ECCD study. We have recently installed an upgraded launcher to extend the ECCD controllability. This paper presents recent experimental results on ECCD using the new launcher system in the Heliotron J. The dependence of ECCD on the parallel refractive index, $N_{\|}$, is investigated. The role of high-energy electrons in ECCD related to magnetic trapping is also discussed. We compare the experimental results with theoretical analysis by using a ray-tracing simulation code, TRAVIS [15]. This paper is organized as follows. The experimental setup including the Heliotron J device and the ECH/ECCD system is described in Sec. 2 . 
In Sec. 3 we present the experimental results, especially the dependence on $N_{\|}$and the magnetic field structure. The experimental results in the Heliotron $\mathrm{J}$ are compared with the results from the TRAVIS code in Sec. 4. A summary is given in Sec. 5.

\section{70-GHz ECH/ECCD System for Heliotron J}

The Heliotron $\mathrm{J}$ is a medium-sized plasma experimental $\mathrm{S} / \mathrm{H}$ device $[16,17]$. The device parameters are: plasma major radius $R=1.2 \mathrm{~m}$, averaged minor radius $a=0.1-0.2 \mathrm{~m}$, rotational transform $\mathrm{t} / 2 \pi=0.3-0.8$ and maximum magnetic field strength on the magnetic field axis $B=1.5 \mathrm{~T}$. The coil system is composed of an $L=1, M=4$ helical coil, two types of toroidal coils A and $\mathrm{B}$, and three pairs of vertical field coils. The configuration is scanned by varying the current ratios in each coil, making it possible to investigate the properties of noninductive current over a wide range of magnetic configurations. Figure 1 illustrates the magnetic field strength along the magnetic axis. The magnetic field ripple is defined by the ratio of the magnetic field at the straight section $(\phi=0 \mathrm{deg})$ to that at the corner section $(\phi= \pm 45 \mathrm{deg}), h=\mathrm{B}_{\text {str }} / \mathrm{B}_{\text {cor }}$. Three configurations, $h=1.06\left(\varepsilon_{\mathrm{b}}=0.01\right), 0.95$ $\left(\varepsilon_{\mathrm{b}}=0.06\right)$ and $0.82\left(\varepsilon_{\mathrm{b}}=0.15\right)$, are chosen. Here $\varepsilon_{\mathrm{b}}$ is defined as $\varepsilon_{\mathrm{b}}=B_{04} / B_{00}$ at the averaged minor radius, $\rho=0.67$, which is varied by controlling the currents in toroidal coils A and B. $B_{\mathrm{mn}}$ is the Fourier component of the magnetic field strength in Boozer coordinates, and $m$ and $n$ are the poloidal and toroidal Fourier harmonic numbers, respectively. The toroidicity $\left(\varepsilon_{\mathrm{t}}=B_{10} / B_{00}\right)$, helicity $\left(\varepsilon_{\mathrm{h}}=\right.$ $\left.B_{14} / B_{00}\right)$, rotational transform and plasma volume are kept almost constant.

Plasmas are produced and heated by $70-\mathrm{GHz}$ second harmonic $\mathrm{X}$-mode $\mathrm{ECH}$, which has a cut-off density of $3.0 \times 10^{19} \mathrm{~m}^{-3}$. We have recently installed an upgraded EC launching system in the Heliotron $\mathrm{J}$ in order to extend the controllability of EC driven current [18]. The upgraded $70-\mathrm{GHz}$ launching system consists of an ellipsoidal mirror and a steerable flat mirror. The steerable mirror enables us to change the beam angle flexibly in the toroidal and poloidal directions. A low power test using a Gunn oscillator shows that the beam radius of $1 / e^{2}$ power is $3 \mathrm{~cm}$ at the magnetic axis, smaller than the minor radius, $a \sim 17 \mathrm{~cm}$, and the available $N_{\|}$ranges from -0.05 to 0.6 , limited by the chamber port size. Here $N_{\|}$is determined by the angle between the resonant magnetic field and EC wave vector under vacuum conditions. The new EC launcher is positioned between the straight and corner sections, while it was positioned at the straight section in the previous experiment, meaning that the power is deposited at a different ripple position. See reference [6] for the previous launching condition. EC beam injection with $N_{\|}=0$ corresponds to injection toward the corner section $(\phi \sim 30$ deg) in the new launcher. For large $N_{\|}$, the EC power is injected toward the straight section direction ( $\phi \sim 15 \mathrm{deg}$ ), and is deposited near the ripple top for $h=1.06$ and near the ripple bottom for $h=0.82$. The X-mode fraction is more than $80 \%$ for the available $N_{\|}$, especially more than $90 \%$ for $0.1<N_{\|}<$ 0.6. The reason why the polarization is not perfect is that only one polarizer mirror is set in the waveguide transmission line due to the limited number of miter bends. The groove parameter is chosen to have an X-mode fraction as high as possible in the available $N_{\|}$range. 


\section{ECCD Experiment}

\subsection{Estimation of EC Driven Current}

Two effects generate EC current in toroidal devices. When EC waves are launched obliquely from low field side in clockwise (CW) toroidal direction, they excite perpendicular excursion in the velocity of a group of electrons with CW parallel velocity. These electrons are accelerated to higher energy and hence to a collisionless location in velocity space. Due to difference in collisional relaxation, the high-energy electrons are symmetrized more slowly than the low-energy electrons, leaving an excess of electrons with $\mathrm{CW}$ velocity, and hence an electrical current in the counter-clockwise (CCW) direction. This is called Fischer-Boozer effect [8]. The other effect is related to a trapping of electrons in velocity space. If the $\mathrm{EC}$ waves move electrons with $\mathrm{CW}$ velocity from the passing region to the trapped region, then the asymmetry of those electrons is lost due to bouncing in the magnetic well on a faster time scale than collision time. The resulting effect of asymmetric trapping and symmetric detrapping creates a deficit in velocity space at the original location of the electrons, generating an electrical current in the $\mathrm{CW}$ toroidal direction. This is called Ohkawa effect. [9] We note that the direction of EC current induced by the Ohkawa effect is opposite to that induced by the Fisch-Boozer effect. The Ohkawa effect is more important as the resonance line is close to the trapping region.

ECCD experiments have been conducted using the upgraded launching system in the Heliotron J. The total toroidal current is measured by Rogowski coils wound on the inner wall of the poloidal cross sections at two different toroidal angles, that is, the corner and the straight sections. An ECH power of $260 \mathrm{~kW}$ is injected up to $140 \mathrm{msec}$. The $L / R$ time is about $100-200 \mathrm{msec}$ for the Heliotron $\mathrm{J}$ plasma parameters, where $L$ and $R$ are the plasma inductance and resistance. In the experiment reported here, the measured toroidal current became saturated within the pulse length for $I_{\mathrm{p}}<3 \mathrm{kA}$.

Figure 2 shows the $N_{\|}$dependence of the measured toroidal current at $\omega_{0} / \omega= \pm 0.499$ at the high $h$ configuration. Here $\omega_{0}$ and $\omega$ are the electron cyclotron frequency at the magnetic axis of the straight section, $\omega_{0}=e B_{\mathrm{st}}(0) / m_{\mathrm{e}}$, and the wave frequency. The electron density is fixed as $\bar{n}_{\mathrm{e}}=0.5 \times 10^{19} \mathrm{~m}^{-}$ ${ }^{3}$. $N_{\|}$is determined at the resonant magnetic field position under vacuum conditions. We define the current flowing in the Fisch-Boozer direction as positive. In ECH plasmas, the toroidal current is composed of the bootstrap current and the EC current. These currents can be separated by comparing the experimental results obtained for $\mathrm{CW}$ and $\mathrm{CCW}$ magnetic fields, since the flow direction of the bootstrap current, which is proportional to the $\mathbf{B} \times \nabla B$ drift, is reversed by reversing the magnetic field, while that of the EC current associated with the $B$ strength is not. The EC driven current, $I_{\mathrm{EC}}$, and the bootstrap current, $I_{\mathrm{BS}}$, are estimated using the equations $I_{\mathrm{EC}}=\left(I_{\mathrm{p}}^{\mathrm{CW}}+I_{\mathrm{p}}^{\mathrm{CCW}}\right) / 2$ and $I_{\mathrm{BS}}=\left(I_{\mathrm{p}}{ }^{\mathrm{CW}}-I_{\mathrm{p}}^{\mathrm{CCW}}\right) / 2$, respectively, where $I_{\mathrm{p}}^{\mathrm{CW}}$ and $I_{\mathrm{p}}^{\mathrm{CCW}}$ are the toroidal currents for the $\mathrm{CW}$ and $\mathrm{CCW}$ magnetic field, respectively. Here we have assumed that the nonlinear interaction between the bootstrap and EC currents is negligible. We confirmed that the other plasma parameters, such as the stored energy, were almost identical in both measurements. In the experiments reported in this paper, the line-averaged electron density measured with a microwave interferometer is $0.2-1.8 \times 10^{19} \mathrm{~m}^{-3}$, and the electron 
temperature measured with a pulse height analysis is $0.3-1.0 \mathrm{keV}$. The electron temperature at $\mathrm{n}_{\mathrm{e}}=$ $0.5 \times 10^{19} \mathrm{~m}^{-3}$ ranges from 0.5 to $0.8 \mathrm{keV}$.

\section{2. $N_{\|}$Dependence of ECCD}

Figure 3 shows the EC driven current as a function of $N_{\|}$in three configurations. The EC driven current numerically calculated by the TRAVIS code (discussed in Sec. 4) is also plotted. Before performing the $N_{\|}$scanning experiment, we adjusted the magnetic field at a fixed $N_{\|}$of 0.4 , to find the magnetic field for the highest EC driven current. For high and medium $h$ configurations, the EC driven current increases with $N_{\|}$in the Fisch-Boozer direction, having maxima of $I_{\mathrm{p}}=2.3 \mathrm{kA}$ and 1.8 $\mathrm{kA}$ at $N_{\|} \sim 0.5$. For the low $h$ configuration $(h=0.82)$, the EC driven current is nearly zero, independent of $N_{\|}$. The estimated bootstrap current is $I_{\mathrm{BS}}=-0.1 \mathrm{kA}, 0.6 \mathrm{kA}$ and $0.53 \mathrm{kA}$ at $N_{\|}=0$ for the configurations of $h=1.06,0.95$ and 0.82 , respectively. These values are close to those derived from neoclassical theory [4]. The reasons for the finite $I_{\mathrm{EC}}$ at $N_{\|}=0$ may be that the off-center EC waves have a finite $N_{\|}$and that the refraction effect makes $N_{\|}$finite. For example, according to the TRAVIS code, the $N_{\|}$of the rays at the peak deposition ranges $-0.06<N_{\|}<0.01$ for $h=0.95$ configuration when $N_{\|}$of central ray is set to be zero. The $N_{\|}$dependence in three configurations indicates that the EC current is more strongly driven when the power is deposited at the high field position in the magnetic ripple structure, and it is suppressed when the EC power is deposited at nearly the bottom of the magnetic field ripple. These EC driven currents are half or less those in the previous experiment (5 $\mathrm{kA}$ and $-2 \mathrm{kA}$ for $h=1.05$ and $h=0.82$, respectively [7]). This may be associated with the difference in EC power deposition position. The EC power was deposited just at either the top or the bottom of the magnetic field ripple in the previous experiment, while the EC power is deposited at in-between position in the experiment reported here (see Fig. 1). Although the achieved EC driven current is reduced, $N_{\|}$control ability is improved by installation of the steerable mirror.

Figure 4 shows the electron cyclotron emission (ECE) signal intensity against $N_{\|}$for three configurations. The ECE signal is measured with a multi-channel radiometer, which views the plasma at the second harmonic frequency near magnetic axis. The ECE intensity is normalized by that at $N_{\|}=$ 0 in each configuration to clarify the configuration dependence. An enhancement of the ECE signals by one order of magnitude and a high correlation between the toroidal current and the ECE intensity have been observed for high $h$ configuration. Since the optical thickness at this low density is gray, $\tau \sim 1$, and the stored energy is weakly changed during the $N_{\|}$scan, the ECE signal reflects not only the bulk $T_{\mathrm{e}}$ but also the high-energy tail. No such enhancement has been observed in the low $h$ configuration where the EC driven current is nearly zero. Although an increase in ECE signals at low density has been also observed in other S/H devices such as W7-AS [19, 20] and L-2M [21], it is not physically clear yet how enhancement of the ECE intensity is connected with the EC driven current. The oblique launch shifts the EC absorption to a higher energy range, increasing the population of high-energy electrons. For low $h$ configuration, the trapped region is so wide that the produced high- 
energy electrons do not move to the other toroidal pitch section, possibly resulting in the radiometer located in a different toroidal section seeing only a few high-energy electrons. Although Soft-X ray spectra measured with the pulse height diagnostic were not available in the $N_{\|}$scanning experiment, an increase in electron energy spectrum in the range of $4-10 \mathrm{keV}$ was observed in the previous experiment when the EC current was driven [7].

\section{Comparison with Theory}

In this section, we compare the experimental results on ECCD in the Heliotron J with a numerical simulation in order to clarify the effect of magnetic field configuration, especially the trapped particle effect. TRAVIS [15] is a ray tracing code for ECH/ECCD and ECE diagnostics in arbitrary 3D magnetic configurations. The code has been applied and benchmarked with some other codes for W7$\mathrm{X}[22,23]$ and ITER $[24,25]$. For ECH/ECCD calculations, the absorption is decomposed into the contributions from trapped and passing electrons by analyzing during integration along the resonance line. The energy range of electrons responsible for absorption is also calculated. The resonant harmonics, which may participate in cyclotron interaction, are defined in the code automatically by analyzing the magnetic configuration. The $\mathrm{CD}$ efficiency is calculated by applying the adjoint approach with parallel momentum conservation (PMC) taken into account [26, 27].

We apply the TRAVIS code to the Heliotron J configurations to calculate the deposition profiles and the ECCD performance. The magnetic configuration provided by the 3D equilibrium code VMEC is converted to Boozer coordinates and interpolated by a highly optimized package. The electron density and temperature profiles are assumed in the simulations to be broad and peaked as $n_{\mathrm{e}}(\rho)=$ $\mathrm{n}_{\mathrm{e}}(0)\left\{0.01+(1-0.01)\left(1-\rho^{8}\right)^{4}\right\}$ and $\mathrm{T}_{\mathrm{e}}(\rho)=\mathrm{T}_{\mathrm{e}}(0)\left\{0.01+(1-0.01)\left(1-\rho^{3}\right)^{1.5}\right\}$, which is typically observed in $\mathrm{ECH}$ plasmas of $\mathrm{S} / \mathrm{H}$ devices. The effective charge is fixed as $Z_{\text {eff }}=2$. Parallel Gaussian beam rays are injected with a beam radius of $3 \mathrm{~cm}$, based on the experimental cold test result. Poloidal and toroidal cross-sections of ray trajectories calculated by TRAVIS code are shown in Fig. 5. The electron density is $n_{\mathrm{e}}(0)=0.5 \times 10^{19} \mathrm{~m}^{-3}$, and the electron temperature is $T_{\mathrm{e}}(0)=0.8 \mathrm{keV}$. The magnetic field is adjusted to locate the power deposition on axis. Figure 6 shows examples of EC power absorption density profiles and EC driven current density profiles in three configurations. $N_{\|}$is set to about 0.4 and the magnetic field strength is adjusted so that the EC driven current can be peaked on the axis. To calculate the efficiency, both the collisional and collisionless limits were applied, which correspond to neglecting and taking into account the trapped electrons, respectively. In the former case, i.e., without the trapped electron effect, the total EC driven current predicted by simulation is $I_{\mathrm{EC}} \sim 12 \mathrm{kA}$, independent of the configuration. This amount of current corresponds to an ECCD efficiency of $\gamma=$ $n_{\mathrm{e}} I_{\mathrm{EC}} R / P_{\mathrm{EC}}=2.4 \times 10^{17} \mathrm{~A} / \mathrm{Wm}^{2}$ and $\zeta=32.7 n_{\mathrm{e}} I_{\mathrm{EC}} R / P_{\mathrm{EC}} T_{\mathrm{e}}=0.10$ where $n_{\mathrm{e}}$ is in units of $10^{20} \mathrm{~m}^{-3}, I_{\mathrm{EC}}$ in $\mathrm{A}, R$ in $\mathrm{m}, P_{\mathrm{EC}}$ in $\mathrm{W}$ and $T_{\mathrm{e}}$ in $\mathrm{keV}$. It is noted that the EC power is not fully absorbed in a single path because of the relatively gray optical depth, $\tau \sim 2$. As a consequence, the EC power is absorbed by electrons not only with $k_{\|} v_{\|}>0$, but also with $k_{\|} v_{\|}<0$, lowering the current drive efficiency as shown in Fig. 7. The main contribution in EC absorption comes from the resonant electrons with velocities 
$v_{\text {res }} / v_{\text {th }}=1.2-2.7$ (here $v_{\text {th }}=\left(2 T_{\mathrm{e}} / m_{\mathrm{e}}\right)^{1 / 2}$ is the thermal velocity). As the EC power is deposited at the ripple bottom (low $h$ configuration), the power fraction absorbed by trapped particles increases: $42 \%$, $52 \%$ and $70 \%$ for $h=1.06,0.95$ and 0.82 , respectively. This leads to a reduction in the EC driven current if the trapped particle effect is included. In the PMC model with the trapped particle effect, the total EC driven current is calculated as 3.0, 1.9 and $-0.4 \mathrm{kA}$ for $h=1.06,0.95$ and 0.82 , respectively. The EC driven current using the PMC model with the trapped particle effect is calculated under the experimental conditions and compared with the experimental results as shown in Fig. 3. The central electron temperature is assumed as $T_{\mathrm{e}}(0)=0.5-0.8 \mathrm{keV}$, based on a soft-X ray pulse height analysis measurement. The TRAVIS calculation reproduces the $N_{\|}$dependence well in all three magnetic configurations considered in the experiments, though there is uncertainty (error bars shown in the theoretical values) in the input parameters such as the $n_{\mathrm{e}}$ and $T_{\mathrm{e}}$ profiles and the effective charge, which have not yet been measured experimentally.

We also compare experimental results obtained using the previous launching system with the TRAVIS code calculation. An unfocused Gaussian beam of 350-kW power was launched from the top of the torus in the straight section where the flux surfaces are bean-shaped and the $B$-contour forms a saddle-type profile. Although the wave beam was injected perpendicular with respect to the equatorial plane, it crossed the resonance layer obliquely because of the 3-D magnetic field structure, resulting in a finite parallel refractive index $N_{\|}$of 0.44 that drove the EC current. Details of the experimental conditions are described in Ref. [4]. Figure 8 shows the $B$ dependence of the EC driven current in three configurations. The central electron temperature is again assumed as $T_{\mathrm{e}}(0)=0.5-0.8 \mathrm{keV}$. The EC power is absorbed on the axis at $\omega_{0} / \omega=0.49$ due to Doppler shift resonance. The numerical results from the TRAVIS code agree with the experimental results and reproduce the configuration dependence. The EC driven current is reduced when the EC power is deposited at the bottom of the magnetic field ripple. The fraction of power absorbed by trapped electrons as a function of magnetic field strength is shown in Fig. 9. The EC driven current in the collisional limit is too large to explain the experimental results, and the trapped particle effect is required to explain the experimental results. The power fraction absorbed by trapped electrons increases with an increase in magnetic field strength, making the EC driven current nearly zero. This tendency is seen also in the other configurations. Figure 10 shows the $n_{\mathrm{e}}$ dependence of the ECCD efficiency, $\gamma$. The experimentally estimated efficiency is insensitive to the electron density, and this dependence is also seen in the simulation. There is discrepancy in the efficiency between experiment and theory by a factor of two, although the physical reason for this is not clear. One of the reasons may be related to experimental accuracy in magnetic field strength. The magnetic field has a saddle-type structure at the EC launching port in the density scan experiment, where the resonant layer position is sensitive to magnetic field strength. In this poloidal cross-section, a few percentage change in the magnetic field strength moves the resonant layer markedly in the core region. The Heliotron J coil system has a setting error in magnetic field about $1 \%$, causing a discrepancy in the efficiency between experiment and theory especially at $\omega_{0} / \omega \sim 0.49$. Experimental data such as $n_{\mathrm{e}}$ and $T_{\mathrm{e}}$ profiles and effective charge 
are also required to measure for more quantitative comparison of the ECCD efficiency.

\section{Summary}

Second-harmonic X-mode ECCD experiments have been conducted in the Heliotron $\mathrm{J}$ using a focused Gaussian beam with a controllable injection angle. The EC driven current was experimentally estimated by excluding the bootstrap current from the total current. The experimental results show that the EC driven current can be controlled by $N_{\|}$and depends on the magnetic configuration. The maximum EC driven current is attained around $N_{\|}=0.5$ when the EC power is deposited nearly at the top of the magnetic ripple, and the EC driven current is nearly zero independent of $N_{\|}$when the EC power is deposited near the bottom of the magnetic ripple. The ECE signal has high correlation with the observed EC current intensity, indicating an important role for high-energy electrons. The experimental results including the $B$ and $N_{\|}$dependences agree with ray tracing simulations using the TRAVIS code in which the parallel momentum conservation with trapped particle effect is considered. Comparing between the collisional and collisionless limit models indicates clear influence of trapped electrons on the ECCD. ECCD may be an effective tool to study the role of rotational transform on confinement and transport such as MHD stabilization and H-mode transition. We will study the effect of localized ECCD on rotational transform profile in the forthcoming experiment. .

\section{Acknowledgments}

The authors are grateful to the Heliotron $\mathrm{J}$ staff for conducting the experiments. This work was performed with the support and under the auspices of the Collaboration Program of the Laboratory for Complex Energy Processes, IAE, Kyoto University, and the NIFS Collaborative Research Program (NIFS08KOAR010, NIFS10KUHL030), the NIFS/NINS project of Formation of International Network for Scientific Collaborations, and a Grant-in-Aid for Scientific Research, MEXT. 


\section{References}

[1] G. Motojima, et al., Plasma and Fusion Res. 3 (2008) S1067.

[2] T. Mizuuchi, et al., Nucl. Fusion 47 (2007) 395.

[3] S. Kobayashi, et al., "Study of spontaneous transition by toroidal current control in a low magnetic shear helical device", 11th IAEA TM on H-mode Phys. Trans. Barriers (Tsukuba, 2007) (http://www-jt60.naka.jaea.go.jp/h-mode-tm-11/index.html).

[4] K. Nagasaki, et al., Nucl. Fusion 45 (2005) 1608-1617.

[5] H. Zohm, et al., Nucl. Fusion 47 (2007) 228-232.

[6] G. Motojima, et al., Nucl. Fusion 47 (2007) 1045.

[7] K. Nagasaki, et al., Nucl. Fusion 50 (2010) 025003.

[8] N. J. Fisch and A. Boozer, Phys. Rev. Lett. 45 (1980) 720.

[9] T. Ohkawa, "Steady state operation of tokamaks by rf heating," General Atomics Report GAA13847 (1976); see National Technical Information Service Document PB2000-108008. Copies may be ordered from NTIS, Springfield, Virginia 22161.

[10]V. Erckmann, et al., Nucl. Fusion 43 (2003) 1313.

[11]A. Fernández, et al., Fusion Sci. Tech. 53 (2008) 254-260.

[12]Y. Yoshimura, et al., Fusion Sci. Tech. 53 (2008) 54-61.

[13]T. Notake, et al., Plasma and Fusion Res. 3 (2008) S1077.

[14]K. Nagasaki, et al., Plasma and Fusion Res. 3 (2008) S1008.

[15]N. B. Marushchenko, et al., Plasma and Fusion Res., 2 (2007) S1129.

[16]M. Wakatani, et al., Nucl. Fusion 40 (2000) 569.

[17]T. Obiki, et al., Nucl. Fusion 41 (2001) 833.

[18]K. Nagasaki, et al., Contrib. Plasma Phys. 50 (2010) 656.

[19]M. Romé, et al., Plasma Phys. Control. Fusion 39 (1997) 117.

[20]N. B. Marushchenko, et al., "On non-local effects of ECE measurements at W7-AS", Proc. 13th Joint Workshop on ECE\& ECRH, Nizhny Novgorod, 17 -20, May, 2004, p. 125.

[21]D. K. Akulina, et al., Plasma Phys. Rep. 32 (2006) 461.

[22]V. Erckmann, et al., Fusion Sci. Technol. 52 (2007) 291.

[23]Yu. Turkin, et al., Proc. 34th EPS Conference on Plasma Phys. Warsaw, 2 - 6 July 2007 ECA Vol.31F, P-1.148 (2007).

[24]N. B. Marushchenko, et al., Nucl. Fusion 48 (2008) 054002;

[25]N. B. Marushchenko, et al., Nucl. Fusion 49 (2009) 129801.

[26]N. B. Marushchenko, et al., Fusion Sci. Technol. 55 (2009) 180.

[27]N. B. Marushchenko, et al., Phys. Plasmas 18 (2011) 032501. 


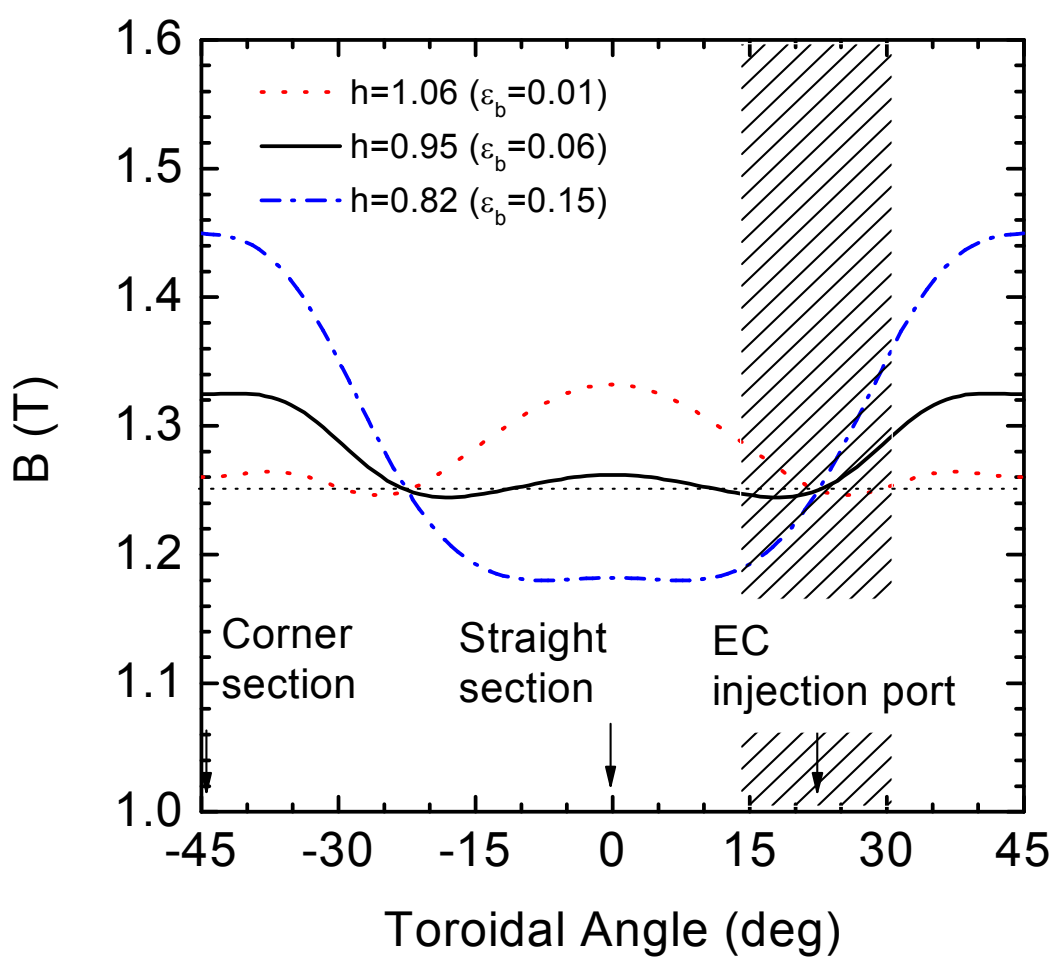

Fig. 1. Magnetic field strength along the magnetic axis for three configurations. The EC power is injected at the chamber port between the straight and corner sections, $\phi=22.5$ deg. The shaded area denotes the available injection range. 


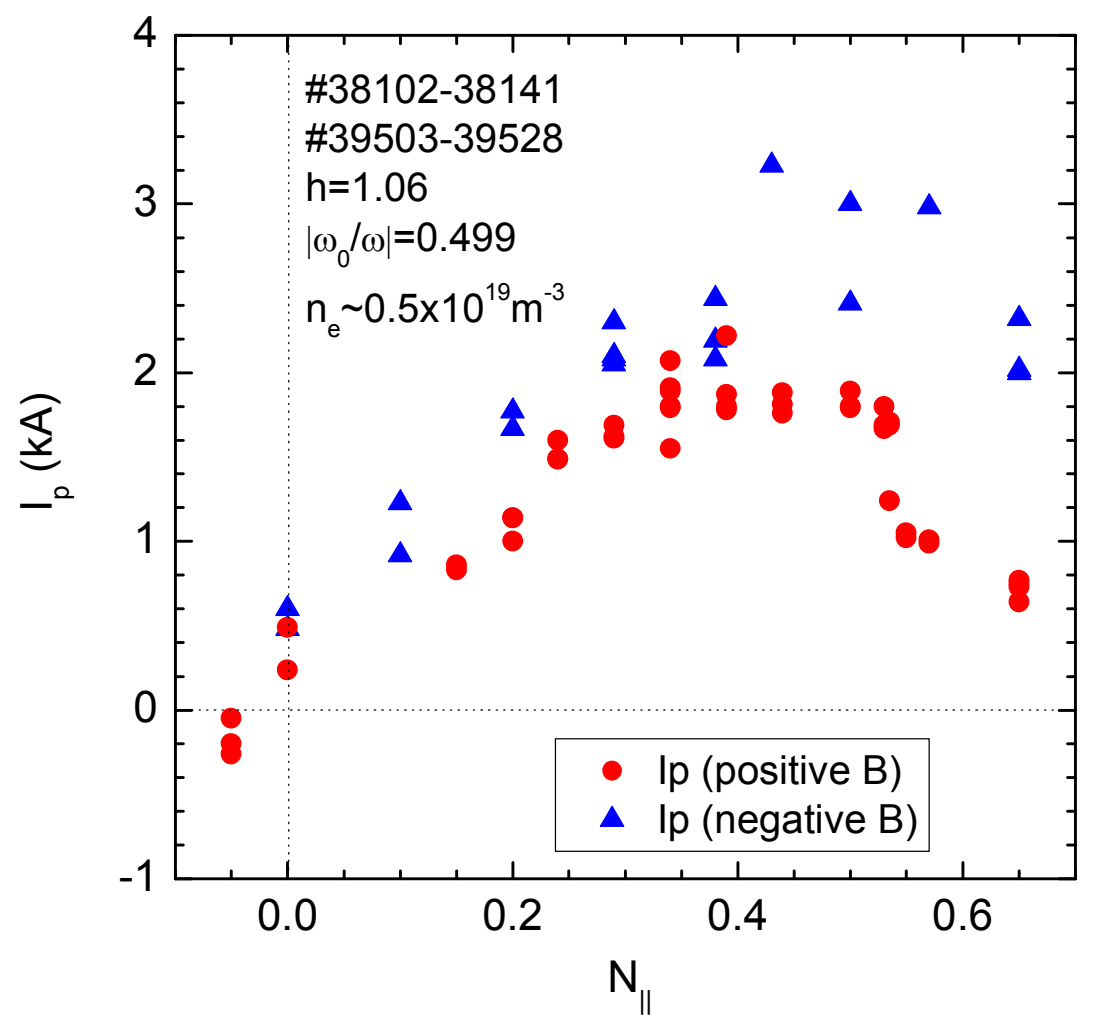

Fig. 2. Measured toroidal current as a function of $N_{\|}$for positive and negative magnetic fields. The injected EC power is $260 \mathrm{~kW}$ and the electron density is fixed as $\bar{n}_{\mathrm{e}} \sim 0.5 \times 10^{19} \mathrm{~m}^{-3}$. 

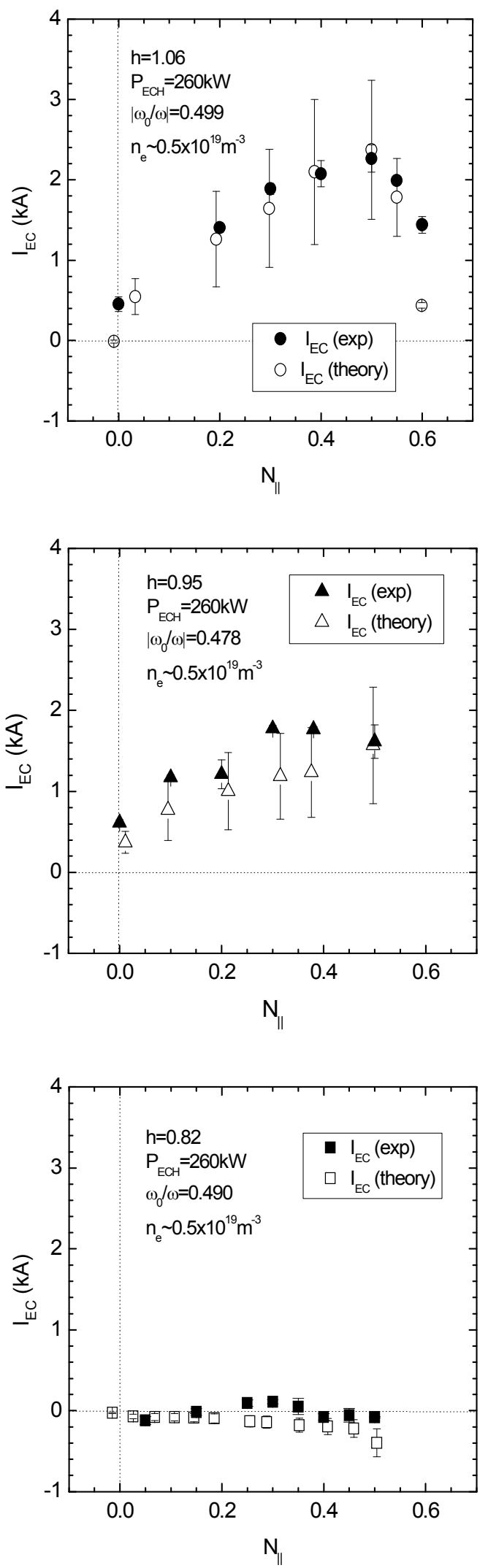

Fig. 3. $N_{\|}$dependence of EC current in three configurations $(h=1.06,0.95$ and 0.82$)$. The closed and open symbols denote the experimental and theoretical EC driven current, respectively. The injected EC power is $260 \mathrm{~kW}$ and the electron density is fixed as $n_{e} \sim 0.5 \times 10^{19} \mathrm{~m}^{-3}$. 


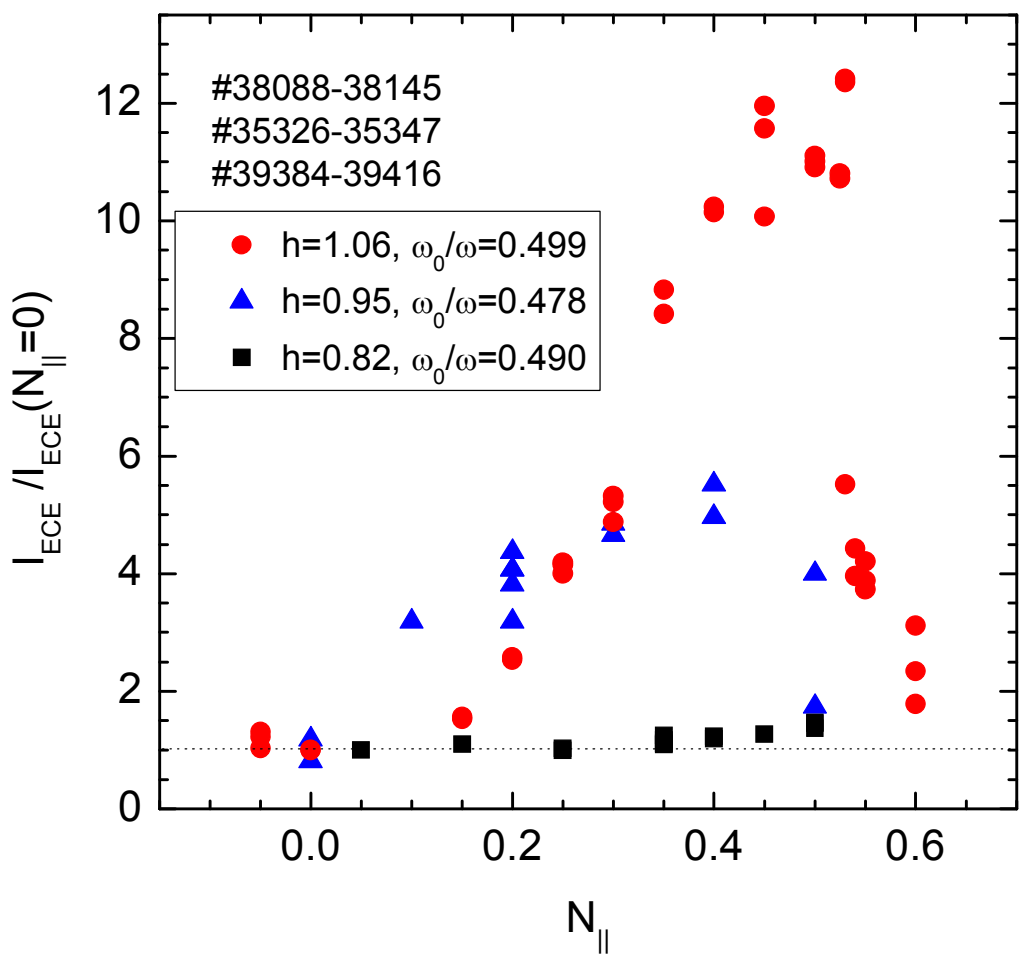

Fig. 4. ECE intensity against $N_{\|}$for three configurations. 

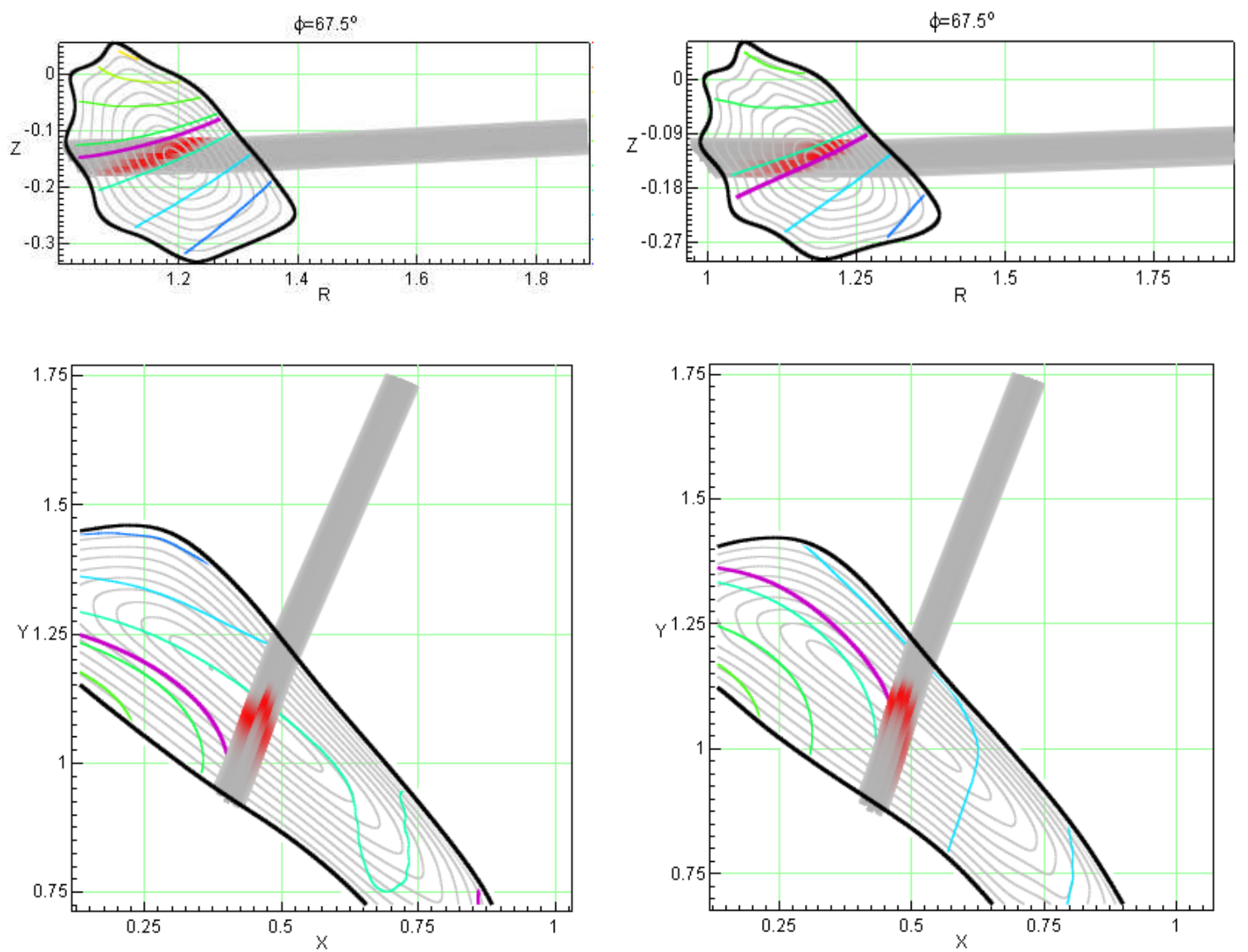

Fig. 5. Poloidal and toroidal cross-sections of ray trajectories calculated by TRAVIS code in two configuration, (a) $h=1.06$ and (b) $h=0.82$. The electron density is $n_{e}(0)=0.5 \times 10^{19} \mathrm{~m}^{-3}$, and the electron temperature is $\mathrm{Te}(0)=0.8 \mathrm{keV}$. The magnetic field is adjusted to locate the power on axis. The bold purple line denotes B=1.25T $\left(\omega_{0} / \omega=0.5\right)$, and the red color denotes the absorbed region. 

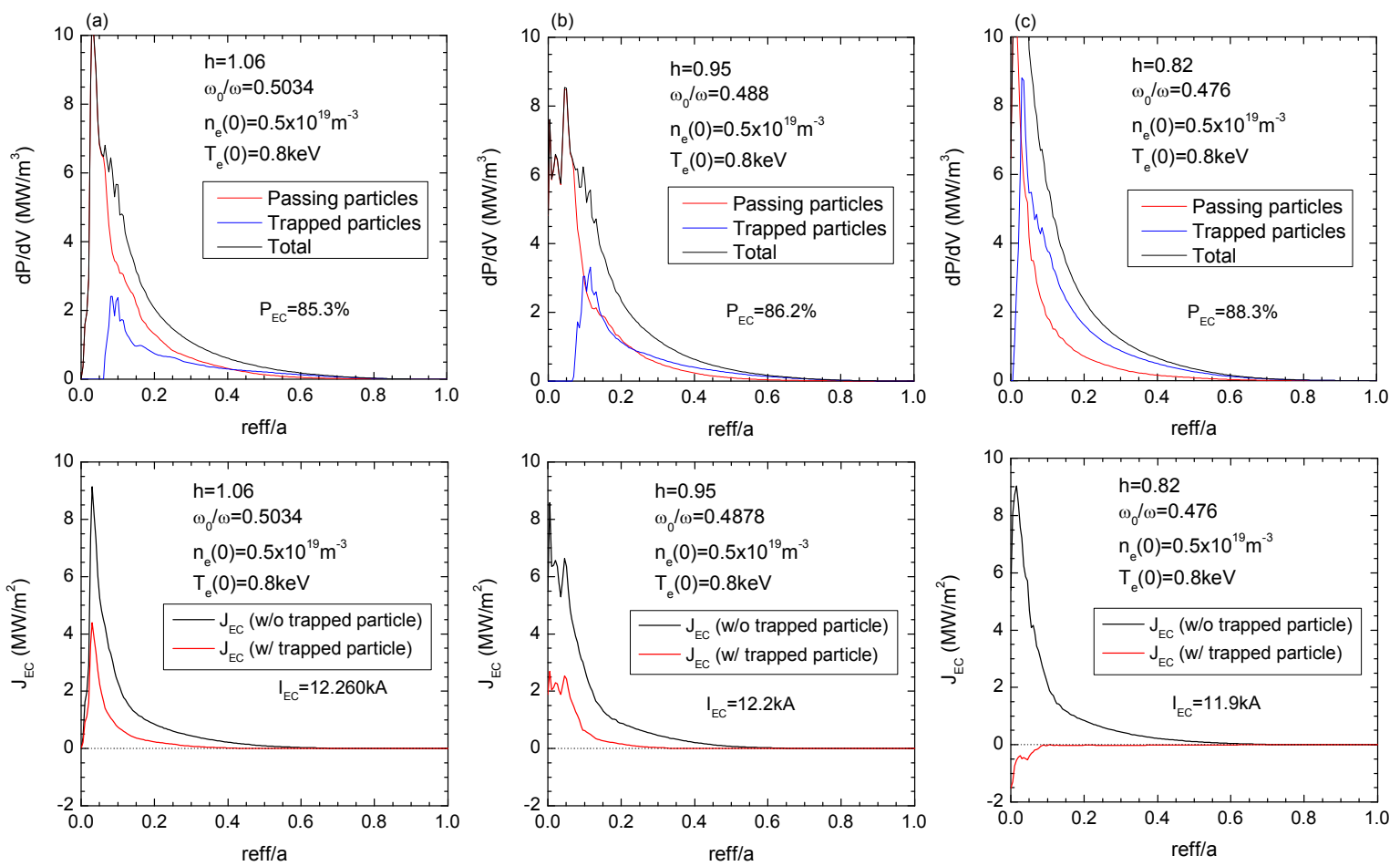

Fig. 6. Power absorption profiles and EC current density profiles calculated by TRAVIS code in three configurations, (a) $h=1.06$, (b) $h=0.95$ and (c) $h=0.82$. The black, red and blue curves denote the total power density and the power density absorbed by passing and trapped particles, respectively. The injected EC power is $350 \mathrm{~kW}$ and the electron density is fixed as $n_{e}(0)=0.5 \times 10^{19} \mathrm{~m}^{-3}$ and the central electron temperature is $0.8 \mathrm{keV}$. 
(a)
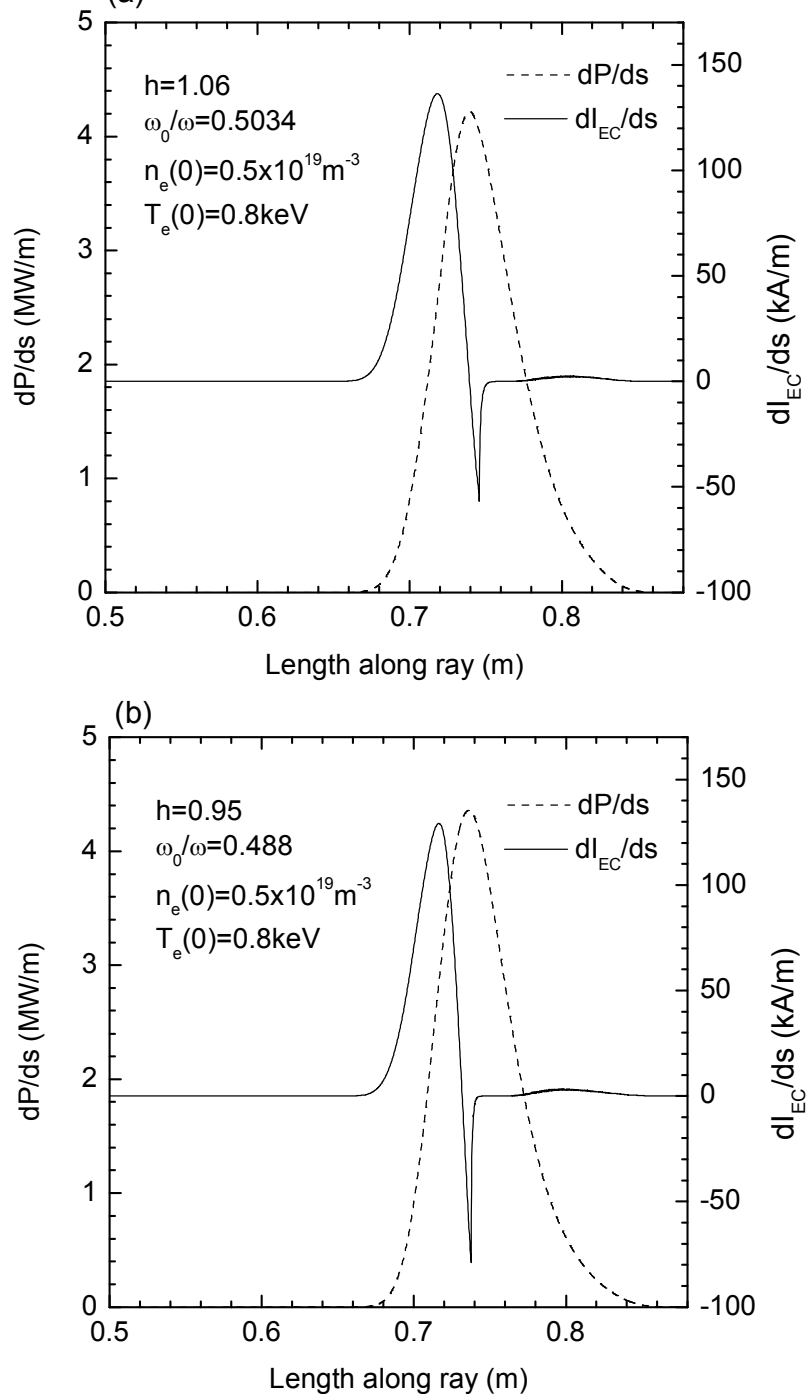

(c)

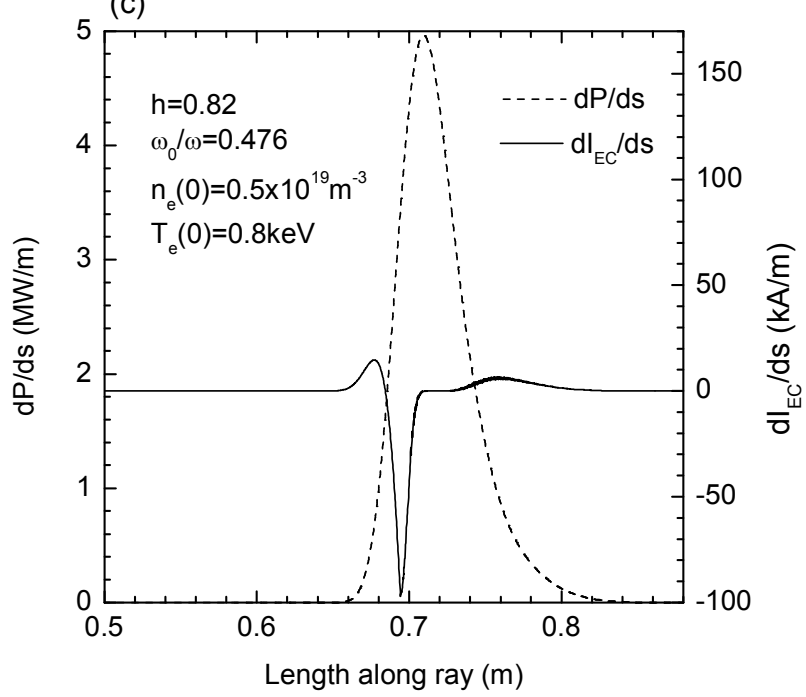

Fig.7. Power absorption and EC current along central ray, calculated by TRAVIS code in three configurations, (a) $h=1.06$, (b) $h=0.95$ and (c) $h=0.82$. The trapped particle effect is included. The input parameters are the same as in Fig. 8. 

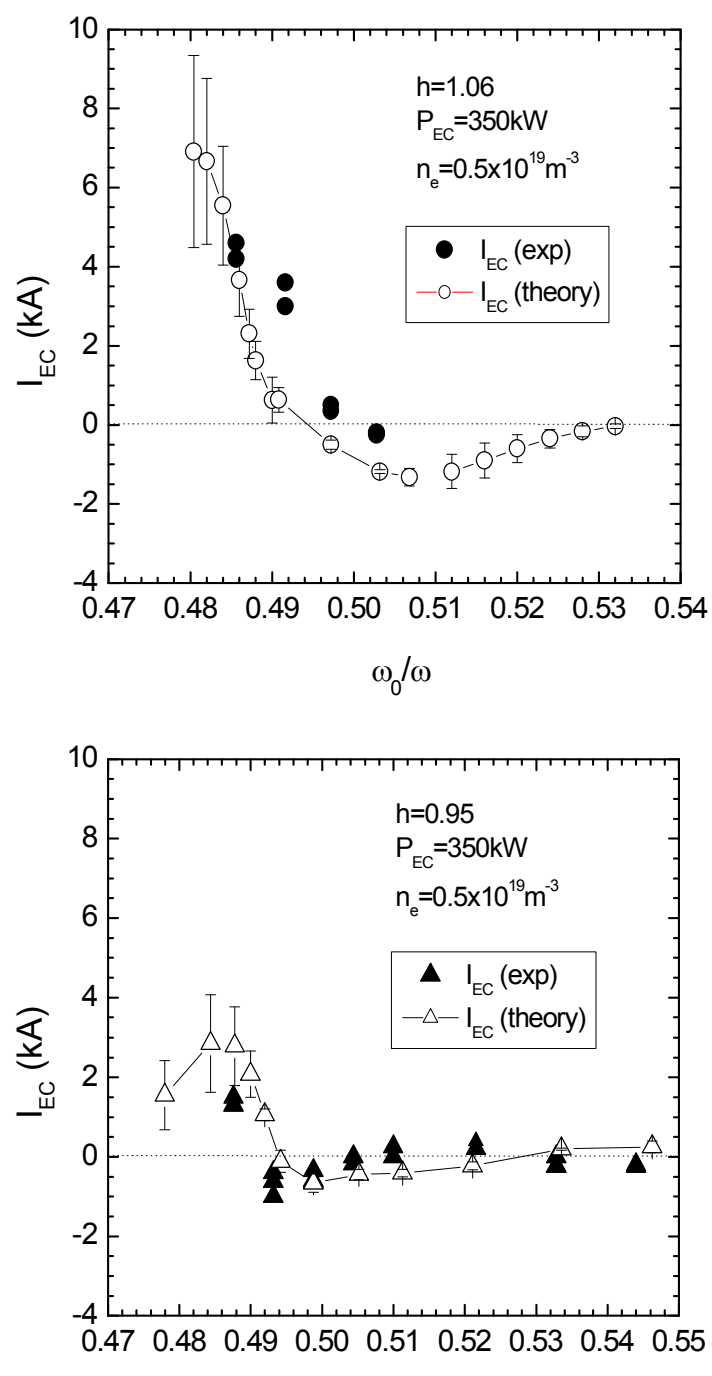

$\omega_{0} / \omega$

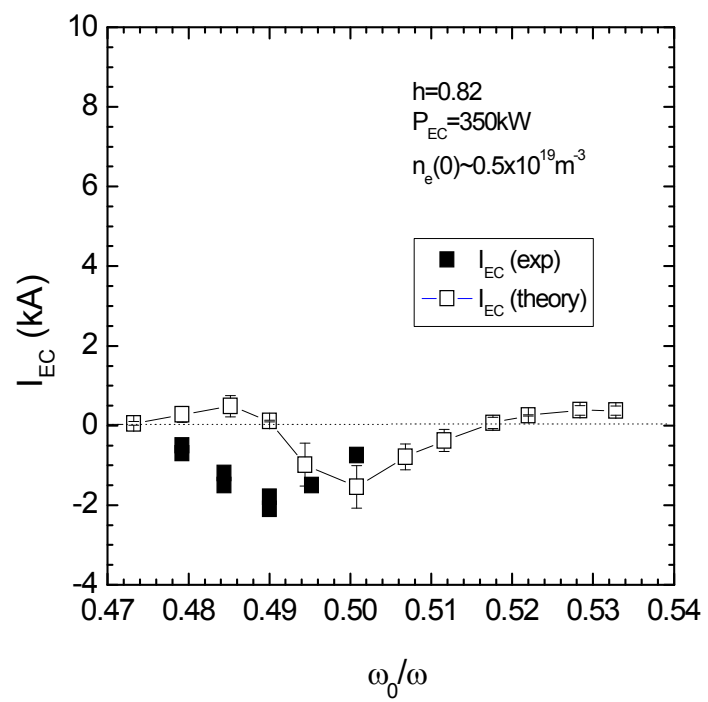

Fig. 8. B dependence of EC driven current in three configurations ( $h=1.06,0.95$ and 0.82). The closed and open symbols denote the experimental and theoretical EC driven currents, respectively. The injected EC power is $350 \mathrm{~kW}$ and the electron density is fixed as $\bar{n}_{\mathrm{e}} \sim 0.5 \times 10^{19} \mathrm{~m}^{-3} . T_{e}(0)$ ranges from 0.5 to $0.8 \mathrm{keV}$ in the TRAVIS calculation. 


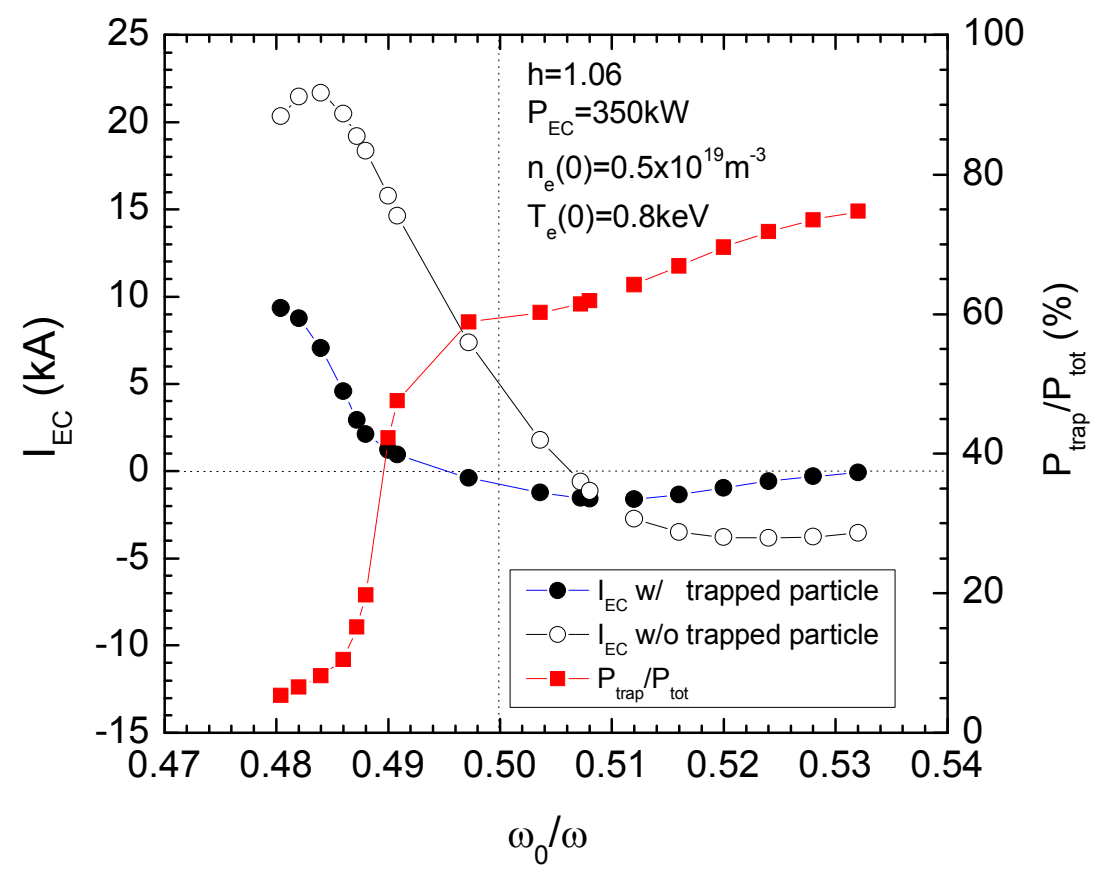

Fig. 9. B dependence of EC driven current calculated by using PMC model in the high $h$ configuration. The power fraction absorbed by trapped particles is also plotted. 


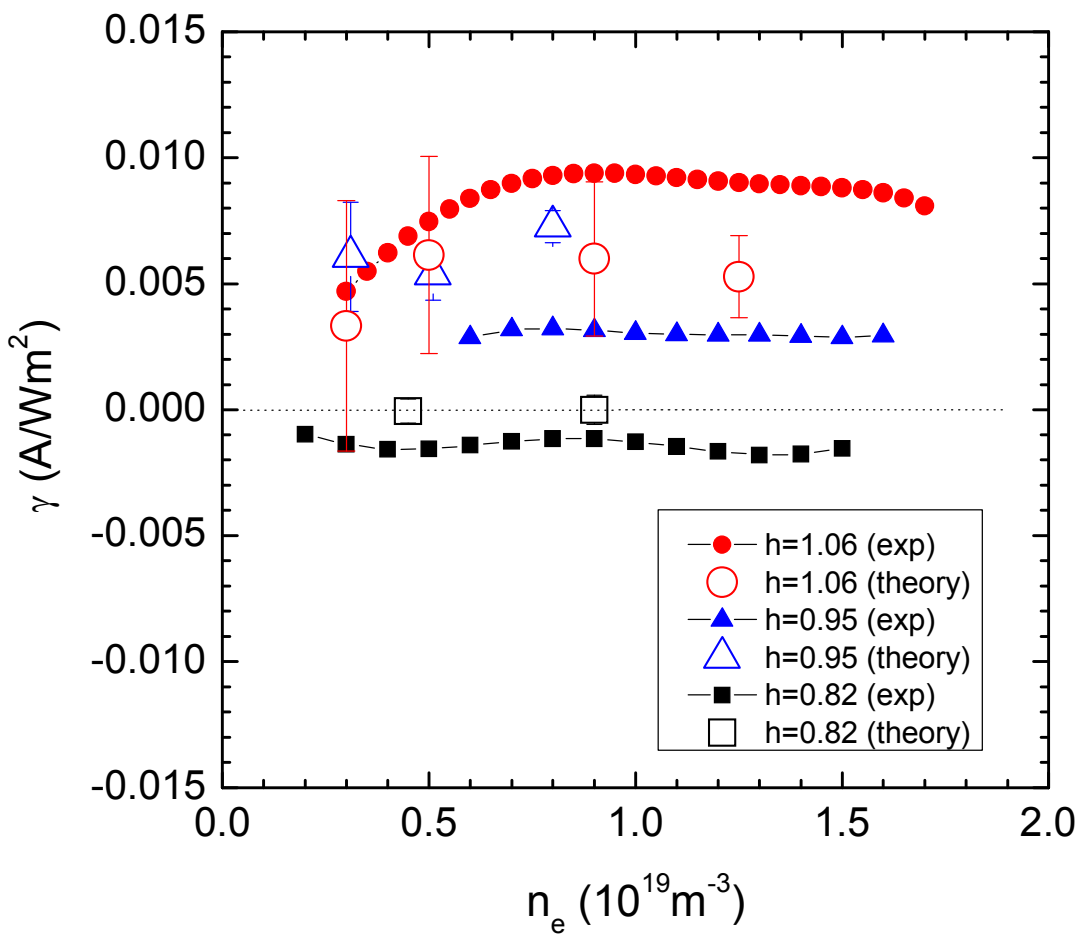

Fig. 10. $n_{e}$ dependence of current drive efficiency, $\gamma=n_{e} R I_{p} / P_{E C}\left(A / W m^{2}\right)$, in three configurations. 